\title{
Expansion of the Central Arborizations of Persistent Sensory Neurons During Insect Metamorphosis: The Role of the Steroid Hormone, 20-Hydroxyecdysone
}

\author{
Richard B. Levine \\ Arizona Research Laboratories Division of Neurobiology, University of Arizona, Tucson, Arizona 85721
}

During insect metamorphosis many larval neurons persist but are modified to serve new behavioral roles at later stages of life. For example, certain larval mechanosensory neurons expand their central arborizations during pupal development and evoke a different behavioral response, the gin trap reflex. The role of the insect steroid hormone, 20-hydroxyecdysone (20-HE) in this developmental change was investigated by removing the normal source of the hormone, followed by topical application of 20-HE to the peripheral somata of the sensory neurons. In prepupal animals that were ligated between the abdomen and thorax to remove the source of ecdysteroids the sensory neurons retained a larval arborization pattern. Topical application of 20-HE to the peripheral sensory neuron somata caused the treated neurons to undergo terminal arbor expansion within the CNS. The treated sensory neurons were not able to evoke the normal pupal behavioral response, but instead caused a larval-like reflex response. In a previous study, sensory neurons that were treated peripherally with a juvenile hormone analog during the commitment peak of ecdysteroids were shown to retain a larval arborization pattern at pupation and to not evoke the gin trap reflex (Levine et al., 1986). Within $4 \mathrm{~d}$ of pupation, however, these neurons belatedly developed expanded terminal arbors and evoked the pupal reflex. In the present study, similarly treated animals were ligated at pupation to block the surge in ecdysteroids that normally occurs at this time. This treatment prevented both the delayed expansion and the reflex, whereas topical 20-HE application induced growth and allowed the treated sensory neurons to evoke the gin trap reflex. It is concluded that both 20-HE and juvenile hormone act directly on the cell bodies of the sensory neurons to regulate the growth of their central processes. This growth is necessary but not sufficient for the development of the gin trap reflex, suggesting that other steroid-dependent changes must also occur within the CNS. Thus, as in the vertebrates, steroid hormones direct important developmental events within the insect nervous system.

Received June 8, 1988; revised Aug. 8, 1988; accepted Aug. 16, 1988.

The author wishes to thank Laura Griffin- $\Lambda$ ustin for her skilled technical assistance and Chip Hedgcock for help with the photography. Dr. Brian Smith provided valuable advice on statistical techniques. Thanks also to Drs. Karla Kent, Brian Waldrop, and Brian Smith for critically evaluating the manuscript. This work was supported by NSF Grant BNS 8607066 .

Correspondence should be addressed to Richard B. Levine, ARL Division of Neurobiology, Room 611, Gould-Simpson Building, University of Arizona, Tucson, AZ 85721

Copyright (C) 1989 Society for Neuroscience $0270-6474 / 89 / 031045-10 \$ 02.00 / 0$
During the process of insect metamorphosis dramatic behavioral changes are accompanied by a reorganization of the nervous system. Although some larval neurons die at the end of the larval period (Weeks and Truman, 1985), and postembryonic neurogenesis contributes to the adult nervous system (Booker and Truman, 1987a), many larval neurons persist during metamorphosis to participate in the generation of adult behavior (Levine and Truman, 1985; Kent and Levine, 1988b). For example, identified motor neurons innervating abdominal body wall muscles of the hawkmoth, Manduca sexta, survive the death of their larval targets and innervate new muscles that are generated during adult development (Levine and Truman, 1985). Similarly, motor neurons innervating the larval thoracic legs of Manduca persist following the degeneration of the larval appendages to innervate muscles in the new adult legs (Kent and Levine, 1988b). In both cases the dendritic arbors of the persistent motor neurons undergo significant remodeling that has been correlated with the acquisition of new synaptic inputs important for adult behavior (Levine and Truman, 1982).

Specific sensory neurons (SNs) also survive beyond the larval stage. For example, the larval abdominal (Levine et al., 1985) and thoracic (Kent and Levine, 1988a) body surfaces are covered with innervated mechanosensory hairs that evoke a weak flexion response when touched (Levine et al., 1985). Although most of the SNs that innervate these hairs die or lose their associated external sensilla at the end of the larval stage, a small number in specific regions of the body surface survive to innervate specialized pupal sensory structures. SNs innervating hairs at the anterior-lateral region of abdominal segments 5-7 survive to innervate hairs within cuticular pits on the pupal surface known as gin traps (Bate, 1973; Levine et al., 1985). Stimulation of these hairs causes a rapid and powerful contraction of the longitudinal muscles of the next anterior segment, bringing the sharp edges of the gin trap in contact with the hard cuticle of the anterior segment. This defensive reflex is unique to the pupal stage of life and is clearly different from the behavior evoked by the same SNs during larval life. Correlated with this behavioral change is the growth by the SNs of extensive new terminal arbors within the CNS (Levine et al., 1985). Thus, differentiated, functional larval neurons are induced to grow new processes and form new synaptic interactions during metamorphosis.

Similar postembryonic changes are known to occur within the vertebrate CNS. For example, thyroxine directs significant changes in the amphibian nervous system during metamorphosis (Hoskins and Grobstein, 1985). Similarly, steroid hormones, which are responsible for the appearance of sex-specific behav- 
ior, induce dramatic changes in the dendritic structure of neurons during postnatal life (DeVoogd and Nottebohm, 1981; Gurney, 1981; Kurz et al., 1986). Hormonal cues also direct the process of insect metamorphosis. The steroid 20-hydroxyecdysone (20-HE) causes cells, such as the epidermal cells that secrete the cuticle, to synthesize new mRNA and protein, while the sesquiterpenoid juvenile hormone $(\mathrm{JH})$ determines which type of protein will be produced (reviewed by Riddiford, 1985). During larval life increases in the blood ecdysteroid titer are accompanied by high levels of $\mathrm{JH}$ and larval cuticle is produced, but at the end of larval life surges in the ecdysteroid titer occur in the absence of $\mathrm{JH}$ and induce the production of pupal cuticular proteins. The small "commitment peak" of ecdysteroids on day 3 of the last larval instar is necessary for the reprogramming of the epidermal cells to a pupal pattern of mRNA synthesis, whereas the larger prepupal peak which starts $2 \mathrm{~d}$ later induces the production of pupal proteins (Riddiford, 1985). Both hormones are believed to act, like vertebrate steroids, by binding to nuclear receptors and regulating gene transcription. It has recently become clear that these 2 hormones also direct metamorphic changes within the insect CNS. At the end of larval life, for example, 20-HE in the normal absence of JH causes the remodeling of motor neuron dendrites (Weeks and Truman, 1985, 1986; Weeks, 1987; Truman and Reiss, 1988).

The gin trap SNs have been useful for exploring the role of $\mathrm{JH}$ and $20-\mathrm{HE}$ in the induction of neural remodeling, and specifically for determining the sites of hormone action within the nervous system. Because the somata of the SNs are located in the periphery, distant from their terminal arbors within the CNS, it is possible to manipulate the hormonal environments experienced by the 2 regions of the cell independently. In an earlier set of experiments it was found that by artificially elevating the JH levels experienced by individual SN somata during the commitment peak of ecdysteroids, the growth of their terminal processes that normally accompanies pupation could be prevented (Levine et al., 1986). Thus, the treated SNs displayed larval branching patterns within the pupal CNS. In such mosaic pupae, untreated pupal SNs evoked the normal gin trap reflex, while JH-treated "larval" SNs did not. It was concluded from this study that the normal decline in $\mathrm{JH}$ is an important signal for the expansion of the SN processes and, furthermore, that the hormonal environment of the cell body of the neuron influences the branching of its terminal processes. These studies also demonstrated that the expanded terminal arbors are necessary for the ability of the SNs to evoke the gin trap reflex.

The previous study did not address the role of $20-\mathrm{HE}$ in the induction of SN terminal growth, nor did it reveal whether information present within the CNS was necessary for correct growth and synapse formation by the SNs. Also not addressed was the possibility that other elements of the reflex circuit (the interneurons and motor neurons) must be modified during the larval-pupal transition. The motor neurons, for example, persist from the larval stage (Levine and Truman, 1985; Levine et al., 1985) and may be modified in response to hormonal cues to participate in the gin trap behavior. In the present study these questions are approached by manipulating the $20-\mathrm{HE}$ environment of the sensory cell bodies. The results indicate that elevated peripheral levels of the steroid in the absence of $\mathrm{JH}$ can induce the growth of SN processes within the CNS but that this growth is not sufficient for the development of the gin trap reflex. Additional steroid-dependent changes are necessary within the CNS. A preliminary account of this work has appeared (Levine, 1986).

\section{Materials and Methods}

Animals. Manduca sexta were reared at $26^{\circ} \mathrm{C}$ under a long-day photoperiod regimen (17 hr light, $7 \mathrm{hr}$ dark) and fed a defined diet (Bell and Joachim, 1978). At the end of the last (fifth) larval instar, the animals ceased to feed and entered the "wandering" stage (see Fig. 1). Pupal ecdysis occurred 4-5 d later. Two populations of animals were used in the present study. Initial experiments (see the 6 groups in Table 2) were performed on animals maintained in a colony established in 1981 at Rice University (Texas), from animals obtained from Texas A\&M University. Later experiments (see the 8 groups in Table 1) were performed on animals maintained in a colony established in 1985 at the University of Arizona, from insects obtained from the USDA laboratories in Beltsville, MD. In all cases where parallel experiments were performed the conclusions were identical for the 2 populations, but because of differences in baseline values (i.e., the arborizations of sensory neurons in the Texas animals were larger) the data were not compared directly between the 2 populations.

Ecdysteroid manipulations. To remove the source of ecdysteroids, larvae (L2) or prepupae (W0, see Fig. 1) were first anesthetized by immersion in tap water or exposure to $\mathrm{CO}_{2}$, then ligated with surgical thread just anterior to the first abdominal segment. The thorax and head were then cut away and discarded. This procedure, which removes both the neurosecretory cells that release the prothoracicotropic hormone and the prothoracic glands that synthesize ecdysone, has been used in several studies to deplete the abdomen of ecdysteroids (e.g., Williams, 1952; Weeks and Truman, 1985; Booker and Truman, 1987b; Morton and Truman, 1988). The ligated abdomens were maintained in the colony incubator and could remain viable for $2-3$ weeks as evidenced by vigorous responses to tactile stimulation, although the experiments reported here were all performed within a week of ligation. In another series of experiments, pupal abdomens were isolated on the day of pupal ecdysis by clamping with a hemostat. The wound was then sealed with warmed petroleum jelly to prevent the loss of hemolymph, but due to the fragile nature of the new pupal cuticle, many animals had to be discarded because of excessive bleeding.

For topical ecdysteroid application it was necessary to mix the highly polar 20-HE in a solution that would allow it to diffuse through the waxy cuticle to the underlying SNs. This was achieved by first dissolving 20-HE (Sigma) in ethanol or isopropanol at a concentration of $1-2 \mathrm{mg} /$ $\mathrm{ml}$. The solution was then mixed with an equal volume of undecane (Sigma). A small droplet of this solution, which covered about $4 \mathrm{~mm}^{2}$ of the body surface after application, was touched to the cuticle in the presumptive gin trap region on one side of abdominal segment 5 of the larva or prepupa. In larvae and prepupae the presumptive gin trap SNs can be distinguished by their characteristic position on the second annulus of abdominal segments 5-7 just dorsal to the spiracle (Bate, 1973; Levine et al., 1985). This procedure was repeated each day for $4 \mathrm{~d}$. Although the steroid concentration in the immediate vicinity of the SNs was impossible to determine due to the probability of incomplete permeation through the cuticle, topical trcatment did not alter the ecdysteroid titer of the general circulation. Four days after ligation, the ecdysteroid level in the hemolymph was quite low (mean, $15.2 \mathrm{ng} / \mathrm{ml} ; n$ $=3$ ). The level was not increased significantly by $4 \mathrm{~d}$ of topical $20-\mathrm{HE}$ application (mean, $16.2 \mathrm{ng} / \mathrm{ml} ; n=3$; ELISA assays performed by Dr. T. Kingan). These values are similar to those measured in normal animals before the commitment and prepupal peaks of ecdysteroid, while the normal ecdysteroid level during the prepupal peak is over $2 \mu \mathrm{g} / \mathrm{ml}$ (Bollenbacher et al., 1981).

$J H$ manipulations. To elevate the $\mathrm{JH}$ titer experienced by individual SNs, a JH analog (JHA, methoprene, Zoecon Corp.) was applied topically (Schneiderman and Gilbert, 1958) to the presumptive gin trap region on day L2-3 as described previously (Levine et al., 1986). The JHA was mixed with soft dental wax at a concentration of $1 \mu \mathrm{g} / \mathrm{ml}$, and a film of the melted mixture was applied to an area ranging from 1 to $8 \mathrm{~mm}^{2}$. The wax solidified immediately and was left in place for $24 \mathrm{hr}$. At the end of this period the wax was removed, and the animal was allowed to complete pupal development.

Staining and measuring of individual sensory neurons. The techniques used for the cobalt staining of individual SNs have been described in detail elsewhere (Levine et al., 1985; Kent and Levine, 1988a). Briefly, a small ring of petroleum jelly was placed around an individual sensory hair and the ring was filled with distilled water. The hair was then plucked out with a pair of fine forceps, the water replaced with a dilute solution of cobalt lysine, and the well covered with more petroleum 


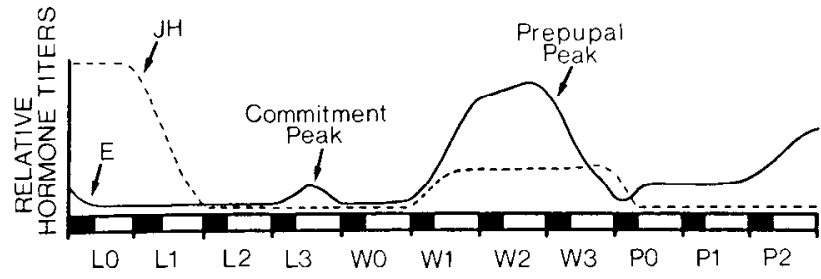

Figure 1. Normal hormone titers during the last (5th) larval instar and early pupal stage of Manduca, (data adapted from Bollenbacher et al., 1981) $L O-L 3$, first $4 \mathrm{~d}$ of the instar; $W 0-W 3$, the first day of wandering and the subsequent $3 \mathrm{~d}$ of the prepupal period; $P 0-P 2$, first $3 \mathrm{~d}$ of the pupal stage. $E$, ecdysteroids; $J H$, juvenile hormone. The black-andwhite bars on the horizontal axis refer to the dark and light photoperiod regimen. In many animals from the Arizona population an extra day was interposed between days $\mathrm{W} 1$ and $\mathrm{P} 0$.

jelly. The preparations were placed in a humid chamber at $4^{\circ} \mathrm{C}$ for $2 \mathrm{~d}$ (early larvae) or $4 \mathrm{~d}$ (prepupae and pupa), after which the nervous systems were dissected out. The preparations were placed in saline that had been saturated with $\mathrm{H}_{2} \mathrm{~S}$, then rinsed in saline, fixed in Carnoy's fixative, silver-intensified, dehydrated in cthanol, cleared in methyl salicylate, and mounted in Canada balsam.

Pupae have gin traps on abdominal segments 5-7. All experiments reported here were performed on SNs in the fifth abdominal segment. Sensory neurons in the gin trap region extend processes into the segmental ganglion within the segment of origin, then course in interganglionic connectives to terminate within the neuropil of the next anterior segmental ganglion. The processes of individual stained SNs were drawn with the aid of a camera lucida attachment to a Zeiss compound microscope. The terminal processes (those within the anterior ganglion) were measured from these 2-dimensional drawings with the aid of a digitizing tablet and Sigma Scan software (Jandel Scientific) run on an IBM AT microcomputer. For each neuron, the total length of all of its processes within the anterior ganglion was termed its "arborization extent."

For sectioning, preparations processed as above were embedded in Epon/araldite (Mollenhauer, 1964; Johnson and Murphey, 1985; Kent and Levine, 1988a) and sectioned with a metal knife at $8 \mu \mathrm{m}$ with a Riechert-Jung model 2040 microtome. The sections were counterstained with toluidine blue and drawn with the aid of a camera lucida attachment.

Behavioral testing. Animals were tested behaviorally for their ability to display the gin trap reflex response by stimulating the sensilla with a fine brush. The normal pupal response consists of a strong, rapid contraction of the ipsilateral intersegmental muscles of the next anterior segment that is terminated quickly (Bate, 1973; Levine et al., 1985). On day P0 (see Fig. 1) stimulation of 1 sensillum within the gin trap is sufficient to evoke a vigorous response. By contrast, early fifth instar larvae typically display no response to stimulation of 1 or 2 sensory hairs within the presumptive gin trap region. Brushing several of the larval sensilla evokes a contraction of the ipsilateral intersegmental muscles in several abdominal segments that is slower, weaker, and more prolonged than the pupal response (Levine et al., 1985). The response magnitude is modulated on days W1-W3 (D. Tamarkin and R. B. Levine, unpublished observations) but is still clearly distinguishable from the pupal behavior because it is multisegmental and sustained.

\section{Results}

\section{Normal growth of sensory neuron terminal arbors during the prepupal period}

The density of sensory hairs in the presumptive gin trap region of the larva does not change as the pupal gin trap forms, although the external structures of the sockets and the hairs are different (Bate, 1973; Levine et al.,1985). During the larval-pupal molt the dendrites of the mechanosensory neurons destined to innervate hairs of the pupal gin trap become detached from the larval sensilla and associated with newly secreted pupal sensilla. In both stages these SNs enter the segmental ganglion via the
Table 1. Effects of ecdysteroids on SN arborization

\begin{tabular}{|c|c|c|}
\hline Group & $\begin{array}{l}\text { Mcan arbori- } \\
\text { zation extent } \\
( \pm 95 \% \text { confid. } \\
\text { limits; } \mu \mathrm{m})\end{array}$ & $n$ \\
\hline Larva $(\mathrm{L} 2)^{*}$ & $342 \pm 103$ & 7 \\
\hline Prepupa (W0)* & $405 \pm 126$ & 4 \\
\hline Pupa (P0) $\ddagger$ & $828 \pm 112$ & 5 \\
\hline W0 ligate $+4 d$ & $431 \pm 36$ & 11 \\
\hline W0 ligate $+4 d+$ solvent & $447 \pm 73$ & 8 \\
\hline W0 ligate $+4 \mathrm{~d}$, untreated side & $418 \pm 49$ & 12 \\
\hline W0 ligate $+4 \mathrm{~d}, 20$-HE treated side $\dagger$ & $649 \pm 30$ & 67 \\
\hline L2 ligate $+4 \mathrm{~d}$, untreated side* & $449 \pm 73$ & 10 \\
\hline L2 ligate $+4 \mathrm{~d}, 20-\mathrm{HE}$ treated side $\dagger$ & $605 \pm 73$ & 10 \\
\hline W3 ligate $+4 \mathrm{~d}+$ & $810 \pm 146$ & 3 \\
\hline
\end{tabular}

All data were from animals reared in the AZ colony. A nonparametric 1-way analysis of variance (Kruskal-Wallis test) indicated that there were significant differences between the 8 groups $(H=76.14, p<0.001)$. The groups fell into 3 statistically differentiated categories (as indicated by the superscripts ${ }^{*}, \dagger, \ddagger$ ), based upon $95 \%$ confidence intervals (see Fig. 2). The means of groups within each category were not significantly different, while the differences between categories were significant. The $3 \mathrm{~W} 0$ control groups enclosed within the bracket were not significantly different from one another, and were grouped together in Figure 2 $(430 \perp 46 \mu \mathrm{m})$.

anterior branch of the dorsal nerve, branch sparsely, and then extend within the interganglionic connectives into the next anterior segmental ganglion before terminating (Levine et al., 1985). It is these anterior processes that have been reported to expand at the end of the larval stage and that were measured in this study. Throughout the study comparisons of the arborization extent values (see Materials and Methods) between the different groups of experimental animals were based upon $95 \%$ confidence intervals. These comparisons indicated that the 8 different groups of treated and control animals within the Arizona set of experiments (see Materials and Methods) fell into 3 significantly different categories (Table 1, Fig. $2 A$ ), there being no significant differences between the means of groups within each of the 3 categories. Similarly, the 6 groups within the Texas set of experiments fell into 3 categories based upon $95 \%$ confidence intervals (Table 2, Fig. $2 B$ ).

\begin{tabular}{lcr}
\hline $\begin{array}{l}\text { Table 2. Effects of ecdysteroids and juvenile hormone on SN } \\
\text { arborization }\end{array}$ & \\
& $\begin{array}{l}\text { Mean arbori- } \\
\text { zation extent } \\
( \pm 95 \% \text { confid. } \\
\text { limits; } \mu \mathrm{m})\end{array}$ & \multicolumn{1}{c}{$n$} \\
\hline Group & $522 \pm 102$ & 6 \\
Larva (L2)* & $1476 \pm 32$ & 16 \\
Pupa (P0) $\ddagger$ & $505 \pm 90$ & 4 \\
W0 ligate $+4 \mathrm{~d}^{*}$ & $1042 \pm 100$ & 28 \\
W0 ligate +4 d, 20-HE treated side† & $676 \pm 250$ & 5 \\
JH on L3, P0 ligate +4 d* & & \\
JH on L3, P0 ligate +4 d, 20-HE & $1118 \pm 200$ & 8 \\
treated side $\dagger$ &
\end{tabular}

All data from animals reared in the TX colony. The groups fell into 3 different caleguries (as indicated by the superscripts *, $\neq, \downarrow$ ), based upon $95 \%$ confidence intervals (see Fig. 2). The means of groups within each category were not significantly different, while the differences between categories were significant. 

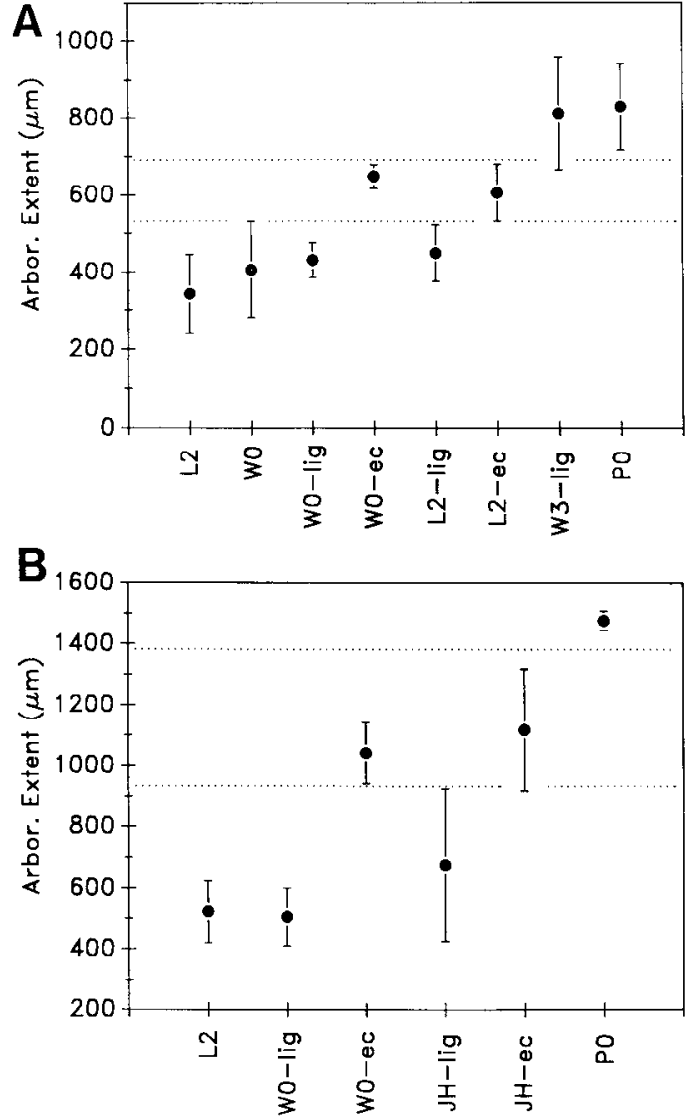

Figure 2. Arborization extent values (mean $\pm 95 \%$ confidence intervals), for different sets of experimental and control animals within the Arizona $(A)$ and the Texas $(B)$ populations. $L 2$, untreated animals from larval day 2; $W 0$, untreated animals from the first day of wandering; W0-lig, animals ligated on day W0 (includes the 3 control groups enclosed within the bracket in Table 1); W0-ec, animals ligated on day W0 and treated topically with 20-HE; L2-lig, animals ligated on day $\mathrm{L} 2 ; L 2-e c$, animals ligated on day $\mathrm{L} 2$ and treated topically with $20-\mathrm{HE}$; W3-lig, animals ligated on day W3; $P 0$, untreated animals from the first day of the pupal stage; JH-lig, animals treated topically with JHA on days L2-L3 and then ligated on day $\mathrm{P} 0 ; \mathrm{JH}-e c$, as previous group but treated topically with $20-\mathrm{HE}$. Note that the different groups can be separated into 3 statistically different categories based upon $95 \%$ confidence intervals (Tables 1 and 2), as indicated by the dotted lines.

In both populations of animals used in the present study the arborization extent of individual SNs in the anterior segmental ganglion was significantly greater in the pupa (P0) than in the larva (L2; Tables 1, 2, Figs. 2-4), as was reported previously (Lcvinc et al., 1985). This increase did not begin until after the onset of wandering. The arborization extent of SNs stained on day W0 was not significantly different from that in prewandering (L2) larvae (Table 1, Fig. 2).

Sections of the anterior ganglia revealed that in both the larval and pupal stages the processes of the SNs were confined to a ventral region of neuropil (Fig. 3), similar to the ventral association center (VAC) described in the segmental ganglia of other insects (Tyrer and Gregory, 1982; Murphey, 1985). The density of branches within this region, however, was greater for pupal SNs. In particular, processes extending into the dorsal portion of the region were more frequent. In addition, the extent of branching in the more anterior regions of the ganglion was greater in the pupal stage.
Preventing the prepupal peak of ecdysone prevents normal growth of sensory arbors

Abdominal ligations on the day of wandering (W0, see Fig. 1) allow the commitment peak of ecdysteroids but prevent the prepupal peak. Abdomens ligated at this time lost their green color but displayed no signs of further pupal development. Four days after ligation, when most normal animals would have pupated, tactile stimulation of the presumptive gin trap hairs did not evoke the characteristic pupal reflex response (sce Matcrials and Methods) but instead caused either no response or a sustained flexion of the entire abdomen, both typical of prepupae (days W0-W3). Furthermore, the arborization extent of SNs stained $4 \mathrm{~d}$ after ligation did not differ from that of prepupal (W0) or larval (L2) neurons (Table 1, Figs. 2, 5), i.e., the SN terminal arbors had not undergone the normal expansion associated with pupation. By contrast, animals that were ligated on day W3 (and therefore experienced most of the prepupal peak of ecdysteroids) formed pupal cuticle within $3 \mathrm{~d}$, as has been reported elsewhere (Weeks, 1987). The gin traps and associated sensilla in these animals were of normal pupal appearance, and tactile stimulation of these sensilla (after removing the overlying larval cuticle) resulted in a normal gin trap response. Sensory neurons in these animals had a mean arborization extent that was not significantly different from that in normal pupae (Table 1, Fig. 2). In another set of W0 animals the effect of nerve cord section alone was tested by cutting the T3-Al connectives, after which the animals were sutured and allowed to pupate. These animals all displayed normal gin trap reflexes on day $\mathrm{P} 0$. Together with the results of the W3 ligation experiments, this suggests that interruption of the nerve cord (which is an unavoidable consequence of the ligation procedure) does not in itself prevent the development of the gin trap reflex.

Exposure of the sensory cell body to 20-HE induces growth of terminal arbors

The failure of sensory processes to expand in ligated animals suggests a role for ecdysteroids in the induction of growth. This possibility was examined in ligated animals by exposing the peripheral somata of presumptive gin trap SNs to elevated ecdysteroid levels. Animals were ligated on day W0. Topical application of the $20-\mathrm{HE} /$ solvent mixture for $4 \mathrm{~d}$ (W0-W3) to the presumptive gin trap region on one side of the fifth abdominal segment caused a significant increase in the arborization extent of the treated SNs compared with normal L2 and W0 animals or to ligated animals in which the SNs had not been treated with 20-HE or had been treated with solvent alone (Figs. 2, 5; Tables 1,2). The arborization extent values in animals treated topically with $20-\mathrm{HE}$ were not, however, as high as those observed in normal pupae (Figs. 2, 5; Tables 1, 2). The influence of 20-HE was specific to the treated SNs and was not due to diffusion of the hormone into the CNS. Individual SNs from the contralateral (control) sides of $20-\mathrm{HE}$ treated animals had an arborization extent that was not different from that of SNs in the other control groups (Table 1). Furthermore, the topical treatment did not increase the ecdysteroid levels in the hemolymph (see Materials and Methods). Therefore, these results suggest that the steroid acts directly upon the peripheral somata of the SNs to induce expansion of their axonal processes within the CNS.

Topical ecdysteroid treatment did not enable the SNs to evoke the gin trap response. Tactile stimulation of the $20-\mathrm{HE}$ treated 


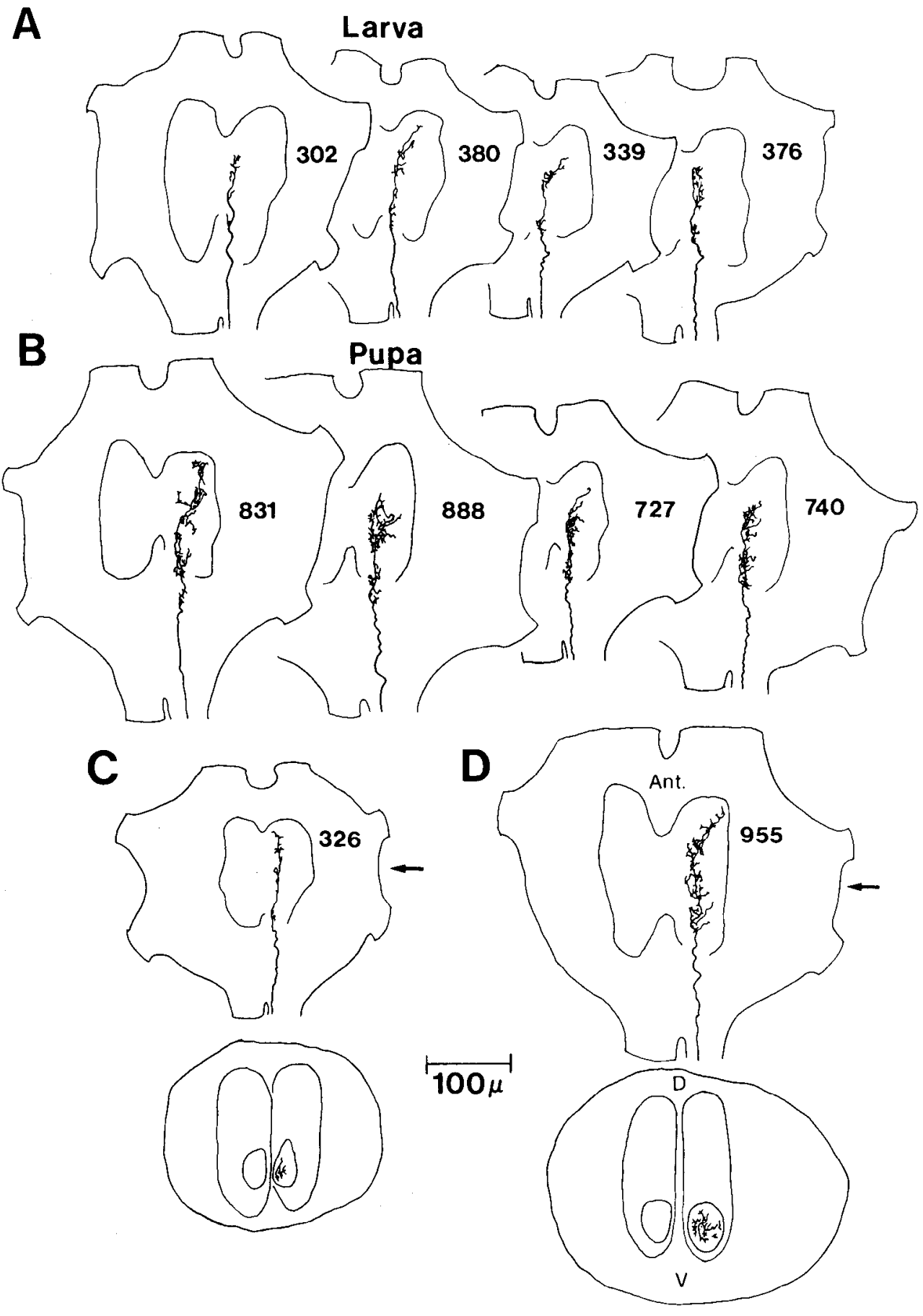

Figure 3. Camera lucida drawings of the terminal arborizations of sensory neurons from the larva $(A, C)$ and the pupa $(B, D)$. In each case the sensory neuron soma was located on the lateral aspect of the 5th abdominal segment, with the terminal processes drawn being located within the 4 th segmental ganglion. Dorsal views are shown in $A$ and $B$ with the "arborization extent" values in microns for cach ncuron indicated within the ganglion outlines. Similar views of a larval and pupal sensory neuron are shown in $C$ and $D$, respectively, with cross-sectional views (composite drawings of four $8 \mu \mathrm{m}$ sections taken at the level of the arrows) shown below. $D$, dorsal; $V$, ventral; Ant., anterior. All preparations from the Arizona population of animals. sensilla evoked the same behavioral response as did stimulation of the untreated side or stimulation of sensilla in normal prepupae, a prolonged weak lateral flexion involving muscles in several segments. This failure to evoke the gin trap response was not due to injury of the SNs. Direct electrical stimulation of the axons of treated SNs also resulted in a prepupal response that could be recorded intracellularly in the intersegmental muscle motor neurons (Tamarkin and Levine, unpublished observations). In addition, in a group of unligated W0 animals an identical series of topical ecdysteroid applications did not prevent the normal expression of the gin trap response after pupation. Therefore, the expansion of terminal arbors that occurs as a result of peripheral 20-HE application to the $\mathrm{SNs}$ does not by itself enable these neurons to evoke the pupal reflex.

The results detailed above indicate that the prepupal pulse of ecdysteroids is necessary for the expansion of the gin trap sensory processes that accompanies pupation. The W0 animals had, however, already experienced the commitment pulse of ecdysteroids at the time of ligation. The normal 2-peak pattern of ecdysteroid exposure is important for some developmental events (Riddiford, 1985), and could be important for the induction of SN growth. For example, the commitment peak (to which the entire animal had been exposed in the experiments described above) might cause changes in the CNS that are required for 
Figure 4. Photos of stained sensory neurons (arrows) from a larva $(A)$ and a pupa $(B)$. In each case, the sensory neuron cell body was in the 5 th abdominal segment, and the terminal axonal processes within the 4 th segmental ganglion are shown with anterior to the top. Scale bar, $100 \mu \mathrm{m}$. Note the greater extent of branching in the pupal preparation.
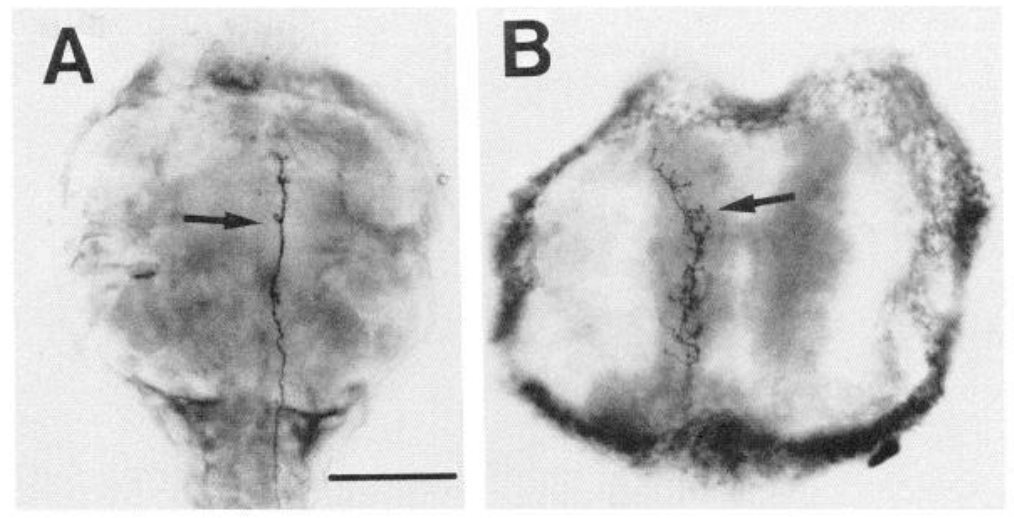

later SN process outgrowth. To examine this possibility animals were ligated on day L2 of the last larval instar to prevent both the prepupal and the commitment peaks of ecdysteroid. Abdomens isolated at this time survived for at least 2 weeks but failed to develop further. The arborization extent of individual SNs from the presumptive gin trap region that were stained 4 $\mathrm{d}$ after ligation did not differ from that of normal larvae (Fig. 2, Table 1). Topical application of 20-HE for $4 \mathrm{~d}$, however, caused the central processes of the treated SNs to increase in extent, although not to the levels seen in normal pupae (Figs. 2, 6, 7; Table 1). Contralateral SNs in the same animals retained a larval arborization pattern, suggesting that the topically ap-
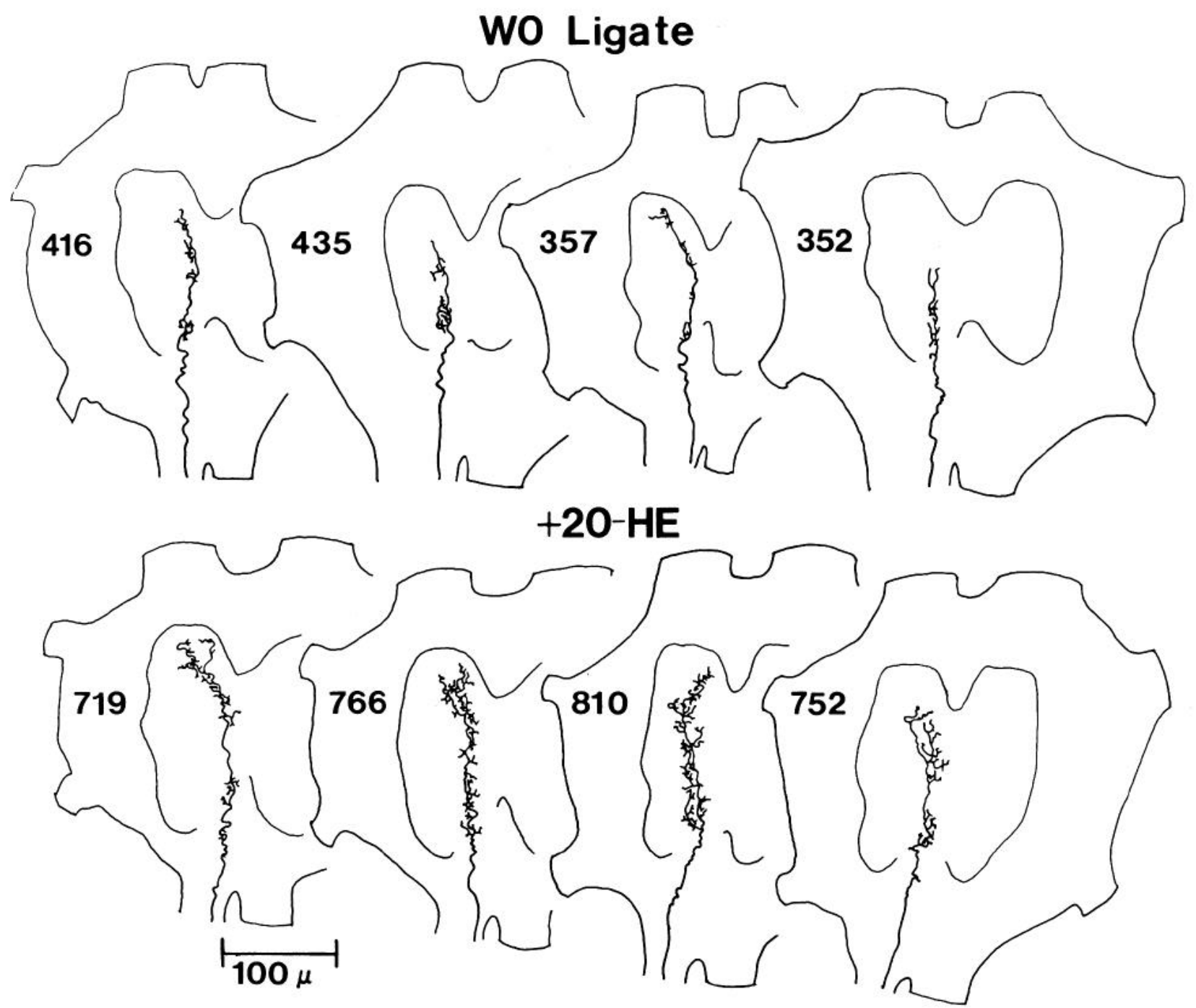

Figure 5. Camera lucida drawings of the terminal axonal processes of sensory neurons in animals ligated on day W0. Those in the lower part of the figure $(+20$-HE) were treated topically with 20 -HE for $4 \mathrm{~d}$ after ligation. Dorsal views are shown with the "arborization extent" value for each neuron given to the left in the ganglion outlines. All preparations from the Arizona population of animals. 


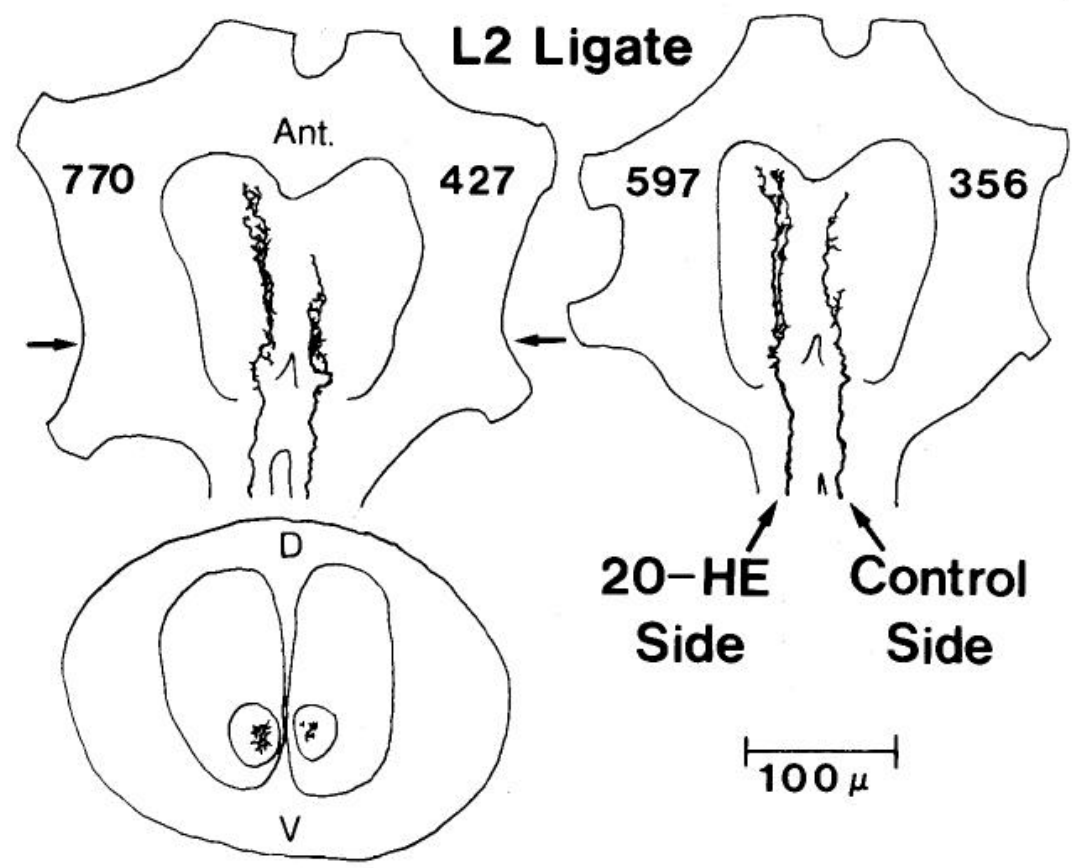

Figure 6. Camera lucida drawings of sensory neuron terminal processes in 2 animals that had been ligated on day L2. In each case the terminals shown on the left side of the ganglion had been treated topically with $20-\mathrm{HE}$ for $4 \mathrm{~d}$ after ligation, while in the same animals the sensory neurons on the right were untreated. Dorsal views are shown at the top with the "arborization extent" values given for the 2 sides. Below (left), cross-sectional view (a composite drawing of four $8 \mu \mathrm{m}$ sections taken at the level of the arrows). $D$, dorsal; $V$, ventral; Ant., anterior.

plied hormone was acting directly on the peripheral somata of the SNs (Figs. 6, 7). The expansion of the SN arbors that resulted from peripheral hormone application to ligated L2 animals was similar in magnitude to that induced by the same hormone treatment of animals that had already experienced the commitment peak. Thus, the ability of topically applied 20 -HE to cause expansion of $\mathrm{SN}$ processes does not require prior exposure of the CNS to the commitment peak of ecdysteroids.

As with animals ligated and treated at later times, growth of the SN central arbors was not linked with the ability to drive the gin trap reflex. Tactile stimulation of treated neurons evoked a weak, prolonged lateral flexion, as is typical of animals at the L2 stage.

\section{Ecdysteroids are responsible for the recovery from $\mathrm{JH}$ treatment}

Topical treatment of presumptive gin trap SNs with JHA during the commitment ecdysteroid peak of the last larval instar caused them to retain their larval branching characteristics within the CNS while the rest of the animal, including untreated SNs, developed normal pupal characteristics (Levine et al., 1986). These JHA-treated SNs failed to evoke the gin trap reflex on the day of pupation, but within 4-5 d they grew normal pupal arbors and attained the ability to evoke the pupal behavior (Levine et al., 1986). It was suggested that the large ecdysteroid surge beginning on day $\mathrm{P} 0$, which normally induces adult development, was responsible for the belated pupal development of the treated SNs. This possibility was tested in the present study by treating larvae topically with JHA during the commitment peak of ecdysteroids to cause the formation of a larval patch of cuticle in place of the gin trap, then ligating the same animals at the time of pupal ecdysis to block the normal increase in ecdysteroid titer.

In such ligated pupae, treated SNs failed to undergo the belated growth of their terminal processes that had been observed in the previous study (Figs. 2, 8; Table 2). Furthermore, whereas the untreated SNs on the contralateral side or in other segments continued to evoke the gin trap response, those that had been exposed to JHA failed to acquire this ability. When the larval patch on the ligated pupae was treated topically with 20 -HE for $4 \mathrm{~d}$, however, the arborization extent of the SNs increased significantly, although not to the normal pupal level (Figs. 2, 8; Table 2). Significantly, topical ecdysteroid treatment did enable the JHA-treated sensory neurons to evoke the gin trap reflex, unlike ecydsteroid-treated SNs in ligated L2 or W0 animals that failed to evoke the pupal behavior.

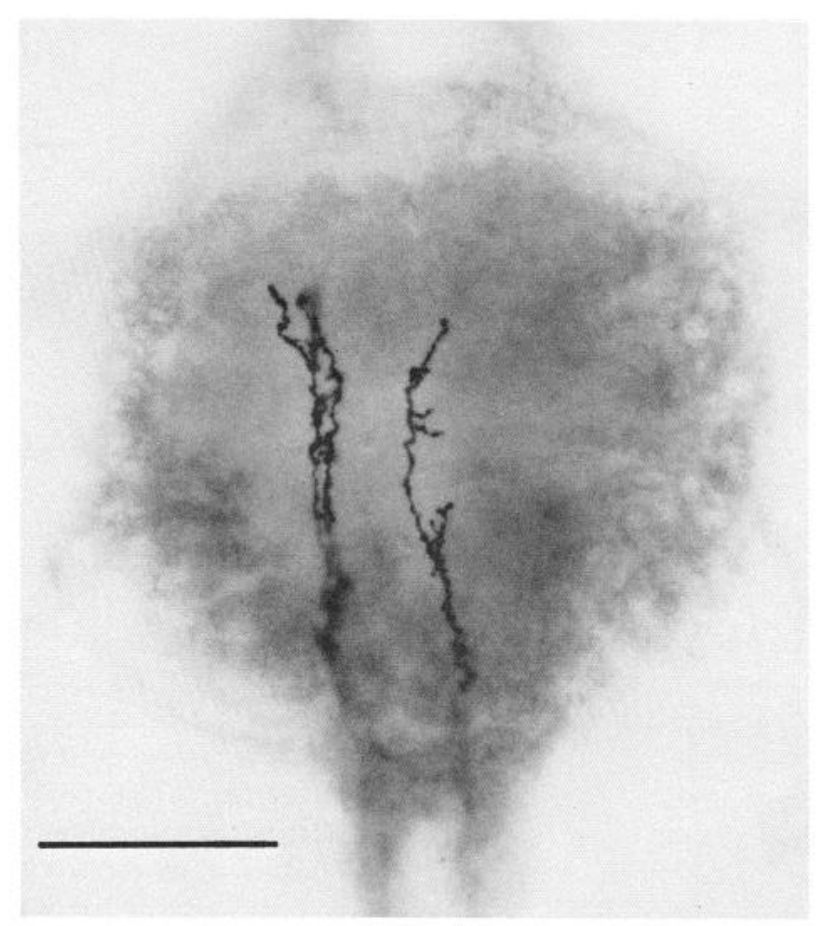

Figure 7. Photo of stained sensory neurons in an animal that had been ligated on day L2. The sensory neuron on the left had been treated topically for $4 \mathrm{~d}$ with 20 -HE. The view is from the dorsal aspect of the ganglion with anterior to the top. Scale bar, $100 \mu \mathrm{m}$. 

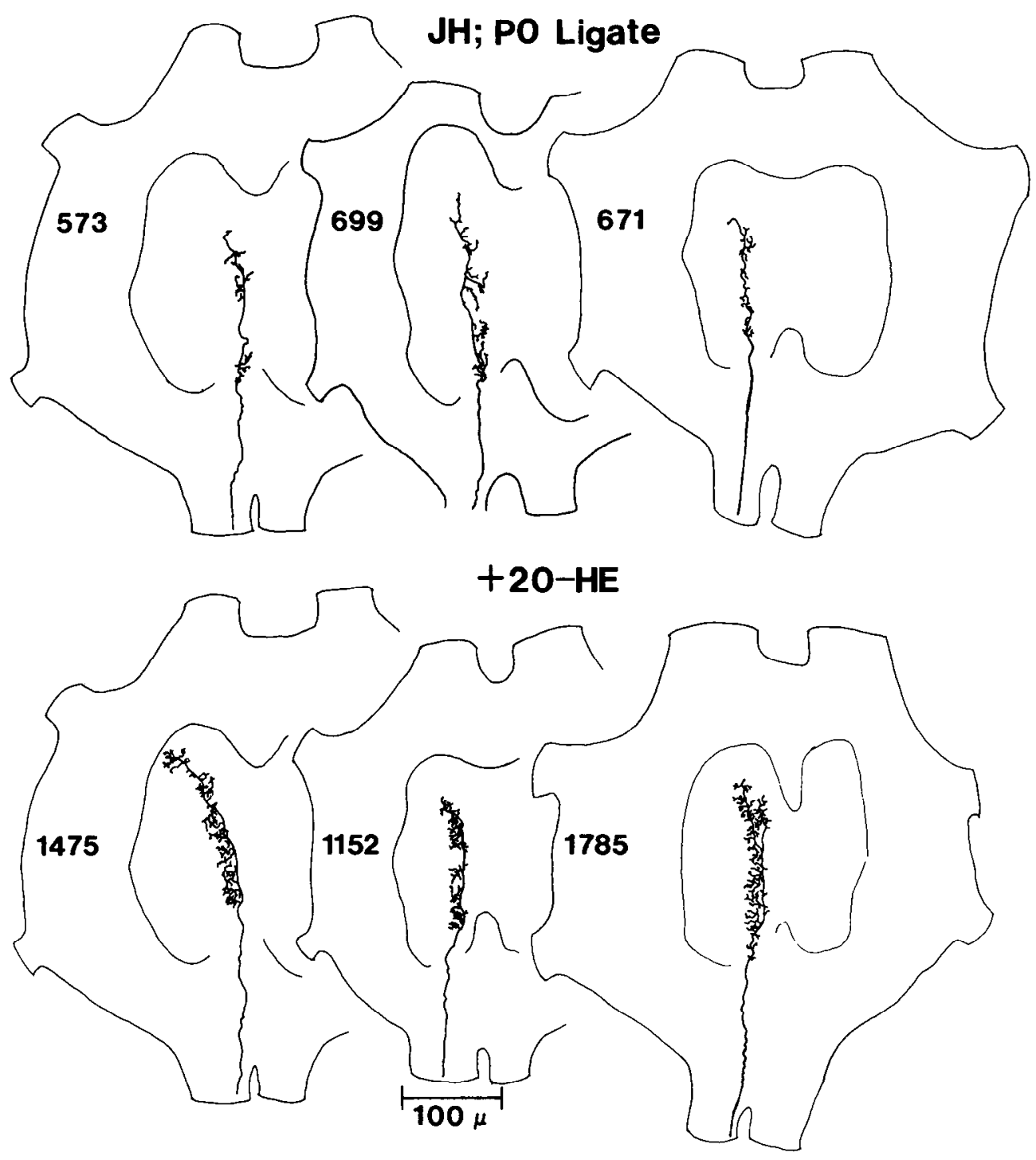

Figure 8. Camera lucida drawings of the terminal arborizations of sensory neurons in animals that had been treated topically with JHA on day L2-L3 to cause formation of a larval patch, then ligated on day P0. Those in the lower series $(+20-H E)$ were treated topically with 20-HE for the first $4 \mathrm{~d}$ of the pupal stage. Dorsal views are shown with anterior to the top. The "arborization extent" values are indicated on the left in each ganglion outline. These preparations were all from the Texas population of animals.

\section{Discussion}

The involvement of $\mathrm{JH}$ and $20-\mathrm{HE}$ in the neuronal reorganization that accompanies insect metamorphosis has now been documented in a number of studies. These hormones regulate cell death (Truman and Schwartz, 1984; Weeks and Truman, 1985,1986 ), the postembryonic differentiation of new neurons (Booker and Truman, 1987b), and the dendritic reorganization and regression of motor neurons (Weeks and Truman, 1985, 1986; Weeks, 1987; Truman and Reiss, 1988). Similarly, the present study, together with an earlier report (Levine et al., 1986), demonstrates that $\mathrm{JH}$ and $20-\mathrm{HE}$ regulate the expansion of the terminal arbors of particular SNs that accompanies pupation. Four lines of evidence support this conclusion. First, abdominal ligation before the prepupal ecdysteroid peak of the last larval instar prevented growth of the sensory arbors. This was true whether or not the abdomens were allowed to experience the commitment peak of ecdysteroids. Second, in such ligated abdomens exposure of the SNs to 20-HE induced expansion of the terminal arborizations of the SNs. Third, exposure of the sensory somata to JHA during the commitment peak of ecdysteroids caused the SNs to retain the larval arborization pattern within the CNS (Levine et al., 1986). There was a critical period during which exogenous JHA application was effective. After the commitment pulse the SNs were insensitive to JHA and underwent normal pupal development. Finally, in animals treated with JHA, belated growth of the sensory arbors was prevented by abdominal ligations in the early pupal stage, which prevented the normal pupal peak of ecdysteroids. In these animals, exposure of the SN somata to 20-HE induced terminal arbor expansion. Together these findings suggest that the gin trap SNs become committed to undergo process outgrowth during the initial exposure to 20 -HE in the absence of JH. They then become independent of $\mathrm{JH}$ and respond to the prepupal ecdysteroid peak by initiating process growth. Unlike other tissues (Riddiford, 1985), the SNs did not require the normal 2- 
pulse pattern of ecdysteroid exposure. Similar results have been reported for the induction of motor neuron death and dendritic regression during the prepupal period (Weeks and Truman, 1986).

The SNs offer one important advantage over other neuron types for the study of steroid-mediated developmental changes. Earlier studies have relied upon infusion of 20-HE into the entire animal to demonstrate an effect of the steroid. With this technique it is impossible to determine which cells are the primary hormone targets. The steroid could be acting directly on the cells of interest or indirectly by affecting other tissues or cells within the CNS. By contrast, the SNs have peripheral somata, which allows their hormonal environment to be manipulated independently from that of the rest of the animal. Thus, exposure of individual SN somata to JHA caused them to retain a larval branching pattern even though the terminal processes were within a pupal CNS that had experienced the hormonal cucs signaling pupal devclopment, and in which ncighboring SNs had formed expanded arborizations (Levine et al., 1986). In the present study, exposure of peripheral SN somata to 20HE caused them to grow more extensive terminal processes even though these processes were within a larval or prepupal CNS that had not experienced an ecdysteroid surge, and in which untreated contralateral SNs retained larval-like arbors. These findings suggest that both $20-\mathrm{HE}$ and $\mathrm{JH}$ act directly upon the SN somata to influence the growth of their terminal axonal processes. Since both $\mathrm{JH}$ and $20-\mathrm{HE}$ are believed to bind to nuclear receptors and regulate gene transcription, it is not surprising that the hormonal environment of the cell bodies should be critical for regulating some features of $\mathrm{SN}$ development. This idea would be supported by the direct demonstration of hormone receptors in the sensory cell nucleus, as has been reported recently for Manduca motor neurons (Fahrbach and Truman, 1987). The results of local hormone manipulation also suggest that steroid-dependent or stage-specific signals within the CNS are neither necessary nor sufficient for the induction of process outgrowth. As discussed below, however, steroid-dependent cues within the CNS may be necessary for directing these new processes to the proper targets or for the formation of correct synapses.

The ability to manipulate the endocrine environment of the SNs independently is valuable for another reason. With the topical manipulation of $\mathrm{JH}$ and $20-\mathrm{HE}$ it has been possible to address the functional rationale for the increased arborization of $\mathrm{SN}$ processes that accompanies pupation. With topical $\mathrm{JH}$ manipulation it was possible to show that SNs that failed to grow new processes could not evoke the gin trap reflex. Since neighboring untreated SNs could evoke a normal pupal response in the same animals, even if they were immediately adjacent to JHA-treated SNs that could not, other elements of the reflex circuit must have been fully competent. This suggests that the increased arborization of the SNs is necessary for the participation of these neurons in the reflex circuit. In the present study, SNs that were induced to grow more extensive terminal arborizations nevertheless failed to evoke the pupal reflex response. Expansion of the SN axonal processes is therefore not sufficient for development of the new behavior.

There are 4 possible reasons why the ecdysteroid-treated SNs failed to evoke the gin trap reflex even though they formed expanded arborizations. First, the topical application may have damaged the sensilla or the SNs may have become detached from the external hairs. This explanation is unlikely since even direct electrical simulation of the sensory axons did not evoke the gin trap reflex (Tamarkin and Levine, unpublished observations). Furthermore, mechanical stimulation of these hairs did evoke a prepupal, or larval-like behavior, proving that the SNs were excited by movement of the hair. Most importantly, topical application of the $20-\mathrm{HE} /$ solvent solution to the presumptive gin trap regions of unligated prepupae did not prevent the formation of a normal gin trap with sensilla that could evoke the typical pupal reflex.

A second possibility is that the level of $20-\mathrm{HE}$ to which the SNs were exposed induced insufficient expansion of the axonal processes or that the processes were not given enough time to grow. Indeed, the arborization extent of the topically treated SNs did not reach that of normal pupae. This explanation seems unsatisfactory, however, since the same dosage of 20-HE and length of time was sufficient to enable SNs in the JHA-treated, ligated pupae to develop the ability to evoke the gin trap reflex, cven though the arborization extent values were less than those in normal pupae. In addition, in the ligated L2 or W0 animals, even those SNs that displayed the most extensive branching increases after $20-\mathrm{HE}$ treatment failed to evoke the gin trap response. To examine this possibility further, higher doses of topically applied $20-\mathrm{HE}$ were tested on the ligated prepupae. These experiments were abandoned, however, because high dosages usually caused significant changes in contralateral SNs, or the development of other pupal characteristics, suggesting that the hemolymph titer of ecdysteroids had been raised significantly. Infusion of 20-HE into ligated prepupal abdomens does lead to relatively normal pupal development (Weeks and Truman, 1986; Morton and Truman, 1988), and the gin traps that form under this condition evoke the normal pupal behavior $(\mathrm{R}$. B. Levine, unpublished observations). Under these conditions, however, the hormone may be influencing many cells within the gin trap circuit in addition to the SNs.

A third possible explanation for the failure of ecdysteroidtreated SNs to evoke the pupal reflex is that peripherally applied 20-HE can induce the SNs to grow new terminal processes but that these processes are unable to find, or form, synapses with their proper targets because these targets have failed to experience a necessary ecdysteroid exposure. The present results do not rule out this possibility. Indeed, the idea is strongly supported by the ability of $20-\mathrm{HE}$ treated SNs in ligated pupae to evoke the gin trap response. In these animals the CNS has, of course, experienced the normal prepupal ecdysteroid titers, unlike animals ligated before pupation. Although it is clear that the new sensory arborizations that were induced by $20-\mathrm{HE}$ application to ligated L2 or W0 animals were in the correct general location within the CNS, a more detailed ultrastructural analysis of the terminal processes will be necessary to determine whether the appropriate synapses have formed. The recent discovery of interneurons that may be involved in the pupal reflex (Waldrop and Levine, 1988) will facilitate this analysis. A related possibility is that the SNs are not the only neurons within the gin trap circuit that undergo a steroid-dependent change during pupal development. For example, the motor neurons that mediate the reflex are retained from the larval stage (Levine and Truman, 1985), and may need to be modified functionally. Similarly, larval interneurons may need to grow new processes or acquire new functional characteristics in response to the hormonal cues that are prevented by ligation.

A final possibility stems from the demonstration that the gin trap circuit must be activated at pupal ecdysis by the peptide eclosion hormone (EH; Levine and Truman, 1983). Two to 3 
hr prior to pupal ecdysis, stimulation of the gin trap SNs normally evokes either no behavioral response or a larval-like response. Injection of EH into intact animals prior to its normal time of release or addition of the peptide to the bath surrounding an isolated nerve cord activated the pupal reflex pathway (Levine and Truman, 1983). Abdominal ligation does not remove the source of this hormone, but recent studies clearly demonstrate that both the release of $\mathrm{EH}$ and the ability of the nervous system to respond to it are under ecdysteroid control (Morton and Truman, 1985, 1988). In summary, it is perhaps not surprising that the expansion of SN central processes is not sufficient for the development of the gin trap reflex. Future studies must investigate the effects of 20 -HE upon other elements of the gin trap circuit, including its ability to modify their response to $\mathrm{EH}$.

In conclusion, these studies support the idea that the steroid hormone 20-HE plays an important role in the metamorphic reorganization of the insect nervous system by directly influencing the morphology of individual neurons. This hormone may also be involved in postembryonic changes that occur within the nervous systems of insects that do not undergo a complete metamorphosis (Chiba et al., 1988). Steroid hormones are responsible for dramatic changes that occur within the vertebrate CNS during postnatal life (DeVoogd and Nottebohm, 1981; Gurney, 1981; Kurz et al., 1986), and further investigation of insect metamorphosis should provide useful insights into the basic mechanisms by which steroid hormones induce such developmental changes.

\section{References}

Bate, C. M. (1973) The mechanism of the pupal gin trap. I. Segmental gradients and the connections of the triggering sensilla. J. Exp. Biol. 59: 95-107.

Bell, R. A., and F. A. Joachim (1978) Techniques for rearing laboratory colonies of the tobacco hornworm and pink bollworms. Ann. Ent. Soc. Am. 69: 365-373.

Bollenbacher, W. E., S. L. Smith, W. Goodman, and L. I. Gilbert (1981) Ecdysteroid titer during the larval-pupal-adult development of the tobacco hornworm, Manduca sexta. Gen. Comp. Endocrinol. 44: 302-306.

Booker, R., and J. W. Truman (1987a) Postembryonic neurogenesis in the CNS of the tobacco hornworm, Manduca sexta. I. Neuroblast arrays and the fate of their progeny during metamorphosis. J. Comp. Neurol. 255: 548-559.

Booker, R., and J. W. Truman (1987b) Postembryonic neurogenesis in the CNS of the tobacco hornworm, Manduca sexta. II. Hormonal control of imaginal nest cell degeneration and differentiation during metamorphosis. J. Neurosci. 7: 4107-4114.

Chiba, A., D. Shepherd, and R. K. Murphey (1988) Synaptic rearrangement during postembryonic development in the cricket. Science 240: $901-906$.

DeVoogd, T. J., and F. Nottebohm (1981) Gonadal hormones induce dendritic growth in the adult avian brain. Science 214: 202-204.

Fahrbach, S. E., and J. W. Truman (1987) Autoradiographic studies of eddysteroid binding in the nervous system of Manduca sexla. Soc. Neurosci. Abstr. 13: 1518.

Gurney, M. E. (1981) Hormonal control of cell form and number in the zebra finch song system. J. Neurosci. 1: 658-673.

Hoskins, S. G., and P. Grobstein (1985) Development of the ipsilateral retinothalamic projection in the frog Xenopus laevis. III. The role of thyroxine. J. Neurosci. 5: 930-940.

Johnson, S. E., and R. K. Murphey (1985) The afferent projection of mesothoracic bristle hairs in the cricket, Acheta domesticus. J. Comp. Physiol. 156: 369-379.

Kent, K. S., and R. B. Levine (1988a) Neural control of leg movements in a metamorphic insect: Sensory and motor elements of the larval thoracic legs in Manduca sexta. J. Comp. Neurol. 271: 559-576.

Kent, K. S., and R. B. Levine (1988b) Neural control of leg movements in a metamorphic insect: Persistence of the larval leg motor neurons to innervate the adult legs of Manduca sexta. J. Comp. Neurol. 276: $30-43$.

Kurz, E. M., D. R. Sengelaub, and A. P. Arnold (1986) Androgens regulate the dendritic length of mammalian motoneurons in adulthood. Science 232: 395-398.

Levine, R. B. (1986) Hormonal control of neuronal reorganization during insect metamorphosis. Soc. Neuro. Abstr. 12: 928.

Levine, R. B., and J. W. Truman (1982) Metamorphosis of the insect nervous system: Changes in the morphology and synaptic interactions of identified cells. Nature 299: 250-252.

Lcvinc, R. B., and J. W. Truman (1983) Peptide activation of a simple neural circuit. Brain. Res. 279: 335-338.

Levine, R. B., and J. W. Truman (1985) Dendritic reorganization of abdominal motoneurons during metamorphosis of the moth, Manduca sexta. J. Neurosci. 5: 2424-2431.

Levine, R. B., C. Pak, and D. Linn (1985) The structure, function and metamorphic reorganization of somatotopically projecting sensory neurons in Manduca sexta larvae. J. Comp. Physiol. 157: 1-13.

Levine, R. B., J. W. Truman, D. Linn, and C. M. Bate (1986) Endocrine regulation of the form and function of axonal arbors during insect metamorphosis. J. Neurosci. 6: 293-299.

Mollenhauer, H. H. (1964) Plastic embedding mixtures for use in electron microscopy. Stain Technol. 39: 111-114.

Morton, D. B., and J. W. Truman (1985) Steroid regulation of the peptide-mediatcd increase in cyclic GMP in the nervous system of the hawkmoth, Manduca sexta. J. Comp. Physiol. A 157: 423-432.

Morton, D. B., and J. W. Truman (1988) The EGPs: The eclosion hormone and cyclic GMP-regulated phosphoproteins. II. Regulation of appearance by the steroid hormone 20-hydroxyecdysone in Manduca sexta. J. Neurosci. 8: 1338-1345.

Murphey, R. K. (1985) A second cricket cercal sensory system: Bristle hairs and the interneurons they activate. J. Comp. Physiol. 156: 357367.

Riddiford, L. M. (1985) Hormone action at the cellular level. In Comprehensive Insect Physiology, Biochemistry, and Pharmacology, Vol. 8, G. A. Kerkut and L. I. Gilbert, eds., pp. 37-84, Pergamon, Oxford.

Schneiderman, H. A., and L. I. Gilbert (1958) Substances with juvenile hormone activity in crustaceans and other invertebrates. Biol. Bull. 115: 530-535.

Truman, J. W., and S. E. Reiss (1988) Hormonal regulation of the shape of identified motoneurons in the moth, Manduca sexta. J. Neurosci. $8: 765-775$.

Truman, J. W., and L. M. Schwartz (1984) Steroid regulation of neuronal death in the moth nervous system. J. Neurosci. 4: 274-280.

Tyrer, N. M., and G. E. Gregory (1982) A guide to the neuroanatomy of locust subesophageal and thoracic ganglia. Phil. Trans. R. Soc London [Biol.] 297: 91-124.

Waldrop, B. R., and R. B. Levine (1988) Interneurons involved in multisegmental reflexes in larvae and pupae of the moth, Manduca sexta. Soc. Neurosci. Abstr. 14: 1003.

Weeks, J. C. (1987) Time course of hormonal independence for developmental events in neurons and other cell types during insect metamorphosis. Dev. Biol. 124: 163-176.

Weeks, J. C., and J. W. Truman (1985) Independent steroid control of the fates of motoneurons and their muscles during insect metamorphosis. J. Neurosci. 5: 2290-2300.

Weeks, J. C., and J. W. Truman (1986) Hormonally mediated reprogramming of muscles and motoneurons during the larval-pupal transformation of the tobacco hornworm, Manduca sexta. J. Exp. Biol. 125: $1-13$.

Williams, C. M. (1952) Physiology of insect diapause. IV: The brain and prothoracic glands as an endocrine system in the Cecropia silkmoth. Biol. Bull. 103: 120-138. 